\title{
$\Gamma$-convergence of Integral Functionals Depending on Vector-valued Functions over Parabolic Domains
}

Huai-Yu Jian

( Department of Mathematics, Tsinghua University, Beijing 100084, P.R. China )

ABSTRACT: We study $\Gamma$-convergence for a sequence of parabolic functionals, $F^{\varepsilon}(u)=\int_{0}^{T} \int_{\Omega} f\left(\frac{x}{\varepsilon}, t, \nabla u\right) d x d t$ as $\varepsilon \rightarrow 0$, where the integrand $f$ is nonconvex, and periodic on the first variable. We obtain the representation formula of the $\Gamma$ limit. Our results in this paper support a conclusion which relates $\Gamma$-convergence of parabolic functionals to the associated gradient flows and confirms one of De Giorgi's conjectures partially.

KEYWORDS: $\Gamma$-convergence, parabolic-minima, nonconvex functionals, parabolic equations.

1991 MR Classification No.: 35B27, 49J45

\section{INTRODUCTION}

We begin with the characterization of $\Gamma$-convergence in $[1,2]$.

DEFINITION 1.1. Let $(X, \tau)$ be a first countable topological space and $\left\{F^{h}\right\}_{h=1}^{\infty}$ be a sequence of functionals from $X$ to $\bar{R}=R \cup\{-\infty, \infty\}, u \in X, \lambda \in \bar{R}$. We call

$$
\lambda=\Gamma(\tau) \lim _{h \rightarrow \infty} F^{h}(u)
$$

if and only if for every sequence $\left\{u^{h}\right\}$ converging to $u$ in $(X, \tau)$

$$
\lambda \leq \liminf _{h \rightarrow \infty} F^{h}\left(u^{h}\right)
$$

and there exists a sequence $\left\{u^{h}\right\}$ converging to $u$ in $(X, \tau)$ such that

$$
\lambda \geq \limsup _{h \rightarrow \infty} F^{h}\left(u^{h}\right) .
$$

We call $\lambda=\Gamma(\tau) \lim _{\varepsilon \rightarrow a} F^{\varepsilon}(u)$ if and only if for every $\varepsilon_{h} \rightarrow a \quad(h \rightarrow \infty)$

$$
\lambda=\Gamma(\tau) \lim _{h \rightarrow \infty} F^{\varepsilon_{h}}(u)
$$

Throughout this paper, we assume that $\Omega$ is a bounded open set in $R^{n}$. Let $p>1, T>0$, and $m$ be a positive integer. Denote

$$
\begin{gathered}
\Omega_{T}=\Omega \times(0, T), \quad V_{p}\left(\Omega_{T}, m\right)=L^{P}\left([0, T], W^{1, p}\left(\Omega, R^{m}\right)\right), \\
V_{p}^{0}\left(\Omega_{T}, m\right)=L^{p}\left([0, T], W_{0}^{1, p}\left(\Omega, R^{m}\right)\right),
\end{gathered}
$$


and

$$
D u(x, t)=\nabla u(x, t)=\left(\frac{\partial u^{i}(x, t)}{\partial x_{j}}\right) \quad(1 \leq i \leq m, 1 \leq j \leq n)
$$

for a vector valued function $u$.

Consider the fuctionals

$$
F_{1}^{\varepsilon}(v, \Omega)=\int_{\Omega} f_{1}\left(\frac{x}{\varepsilon}, D v\right) d x, \quad v \in W^{1, p}\left(\Omega, R^{m}\right),\left(\varepsilon \rightarrow 0^{+}\right)
$$

and the corresponding parabolic functionals in the following form:

$$
F^{\varepsilon}\left(u, \Omega_{T}\right)=\int_{\Omega_{T}} f\left(\frac{x}{\varepsilon}, t, D u\right) d x d t, \quad u \in V_{p}\left(\Omega_{T}, m\right),\left(\varepsilon \rightarrow 0^{+}\right),
$$

where $f: R^{n+1} \times R^{m n} \rightarrow R$ is a Caratheodory function satsfying

$$
C_{1}|\lambda|^{p} \leq f(x, t, \lambda) \leq C_{2}\left(1+|\lambda|^{p}\right)
$$

for some positive constants $C_{2}>C_{1}$.

In 1979, E. De Giorgi [3] conjectured that when a sequence of functionals, for instance, the one in (1.4) or in a more general form, converges in the sence of $\Gamma$-convergence to a limiting functional, the corresponding gradient flows will converge as well (maybe after an appropriate change of timescale). Also [4, p.216] and $[5$, p.507].

In [6], the author proved the De Giorgi's conjecture for a rather wide kind of functionals. Thus, a natural question is that under what conditions, the functional sequence like (1.4) can be $\Gamma$-convergence.

A first result related to this question was appeared in [7]. Because the integrands in [7] have the same scale for the variables $x$ and $t$, the methods there can't be applied to functionals (1.4) whose integrands are anisotropic in $x$ and $t$.

In this paper, we will cleverly combine the arguements in $[8,9,10]$, all of which study the $\Gamma$-convergence of elliptic functionals like (1.3) with the weak-topology of $W^{1, p}\left(\Omega, R^{m}\right)$, to prove that the $\Gamma$-convergence holds for the functional (1.4) under assumption (1.5) and a periodic hypothesis (see (1.8) below). For this purpose, we construct functionals as follows.

Let $Y=(0,1)^{n}=\left\{0<y_{i}<1, i=1,2, \cdots n\right\}, k Y=(0, k)^{n}$, and $k_{T}=$ $k Y \times(0, T)$. For $\lambda \in R^{m n}$ and a.e. $t \in R$, define

$$
\bar{f}(t, \lambda)=\inf _{k \in N} \inf \left\{|k Y|^{-1} \int_{k Y} f(y, t, \lambda+D \phi(y, t)) d y: \phi \in V_{p}^{0}\left(k_{T}, m\right)\right\},
$$

where and below $|E| \stackrel{\text { def }}{=} L^{n}(E)$ and $L^{k}$ is used to denote the $k$-dimensional Lebesque measure. 
Obviously (1.5) implies that $\bar{f}(t, D u)$ is nonnegative and measurable, so we can define the homogenized functional

$$
F\left(u, \Omega_{T}\right)=\int_{\Omega_{T}} \bar{f}(t, D u) d x d t, \quad u \in V_{p}\left(\Omega_{T}, m\right) .
$$

The main result of this paper is the following theorem.

THEOREM 1.2. If hypotheses (1.4) and (1.5) are satisfied, and suppose

$$
f(y, t, \lambda) \text { is } \bar{Y} \text {-periodic on the first variable } y
$$

then for every $T>0$ and every bounded open set $\Omega \subset R^{n}$ with $L^{n}(\partial \Omega)=0$

$$
\Gamma(\tau) \lim _{\varepsilon \rightarrow 0} F^{\varepsilon}\left(u, \Omega_{T}\right)=F\left(u, \Omega_{T}\right), \forall u \in V_{p}\left(\Omega_{T}, m\right),
$$

where $\tau$ is taken as the sw-topology of $V_{p}\left(\Omega_{T}, m\right)$. ( See the Def. 1.2 in [6] for the sw-topology.

The proof of this theorem will be given in section 4 .

\section{PRELIMINARY LEMMAS}

We collect some properties of the $\Gamma$-limits in $[1,2]$ which are well-known but important for the coming arguements.

If the limsup in (1.2) is replaced by liminf, the definition 1.1 is turned to the definition of low $\Gamma$-limit. In this case, we denote it by

$$
\lambda=\Gamma^{-}(\tau) \lim _{h \rightarrow \infty} F^{h}(u) .
$$

Similarly, we have upper $\Gamma$-limit and denote it by $\lambda=\Gamma^{+}(\tau) \lim _{h \rightarrow \infty} F^{h}(u)$.

Obviously, $\Gamma(\tau) \lim _{h \rightarrow \infty} F^{h}(u)$ exists if amd only if $\Gamma^{+}(\tau) \lim _{h \rightarrow \infty} F^{h}(u)=$ $\Gamma^{-}(\tau) \lim _{h \rightarrow \infty} F^{h}(u)$.

LEMMA 2.1. $F^{-}(u)=\Gamma^{-}(\tau) \lim _{h \rightarrow \infty} F^{h}(u)$ exists for every $u \in X$, and $F^{-}(u)$ is lower semicontinuous in $(X, \tau)$. If $F(u)=\Gamma(\tau) \lim _{h \rightarrow \infty} F^{h}(u)$ exists for every $u \in X$, then $F(u)$ is also lower semicontinuous in $(X, \tau)$.

LEMMA 2.2. For each sequence $\left\{F^{h}\right\}$ of functionals in $(X, \tau)$, there exists a subsequence $F^{h_{k}}$ and $F^{\infty}$ from $X$ to $\bar{R}$, such that

$$
F^{\infty}(u)=\Gamma(\tau) \lim _{k \rightarrow \infty} F^{h_{k}}(u) \quad \forall u \in X
$$

LEMMA 2.3. Suppose that $\lambda=\Gamma(\tau) \lim _{\varepsilon \rightarrow 0} F^{\varepsilon}(u)$ and $\varepsilon_{h} \rightarrow 0(h \rightarrow \infty)$, then

$$
\Gamma^{-}(\tau) \lim _{h \rightarrow \infty} F^{\varepsilon_{h}}(u)=\Gamma(\tau) \lim _{h \rightarrow \infty} F^{\varepsilon_{h}}(u)=\lambda
$$


LEMMA 2.4. Suppose that $f: R \times R \rightarrow \bar{R}$, then there exists a function $\delta: \varepsilon \rightarrow \delta(\varepsilon)$ such that $\varepsilon \rightarrow 0$ implies $\delta(\varepsilon) \rightarrow 0$ and

$$
\limsup _{\varepsilon \rightarrow 0} f(\delta(\varepsilon), \varepsilon) \leq \limsup _{\delta \rightarrow 0} \limsup _{\varepsilon \rightarrow 0} f(\delta, \varepsilon)
$$

Moreover, the opposite inequality for low limits and the equality for limits hold true respectively.

From now on, we restrict ourselves to the sequence of functionals (1.4), or more general functionals :

$$
F^{\varepsilon}(u, \Omega \times(a, b))=\int_{a}^{b} \int_{\Omega} f\left(\frac{x}{\varepsilon}, t, D u\right) d x d t, \quad\left(\varepsilon \rightarrow 0^{+}\right) .
$$

We will fix $T>0$ and allow $\Omega$ and $(a, b)$ to be arbitrary. Let $S=R^{n} \times(0, T)$, $\beta_{T}$ be the $\sigma$-ring generated by the set

$$
\left\{\Omega \times(a, b): 0 \leq a<b \leq T, \Omega \subset R^{n} \text { are bounded open sets }\right\} .
$$

Then $\left(S, \beta_{T}, L^{n+1}\right)$ is a measure space. Let

$$
V_{p, l o c}=L^{p}\left([0, T], W_{l o c}^{1, p}\left(R^{n}, R^{m}\right)\right)
$$

LEMMA 2.5. Assume that (1.4), (1.5) and (1.8) are satisfied. Then for every sequence $\varepsilon \rightarrow 0^{+}$, there exist a subsequence $\varepsilon_{h} \rightarrow 0^{+} \quad(h \rightarrow 0)$ and a family of $\sigma$-finite and $\sigma$-additive measures $H(u, \Omega \times(a, b))$ on $\beta_{T}$, such that for every $u \in V_{p, l o c}$, every finite interval $(a, b)$ and every bounded open set $\Omega \subset R^{n}$ with $L^{n}(\partial \Omega)=0$,

$$
\Gamma(\tau) \lim _{h \rightarrow \infty} F^{\varepsilon_{h}}(u, \Omega \times(a, b))=H(u, \Omega \times(a, b))
$$

and

$$
0 \leq H(u, \Omega \times(a, b)) \leq C \int_{a}^{b} \int_{\Omega}\left(1+|D u|^{p}\right) d x d t,
$$

where $\tau$ is the sw-topology of $V_{p}(\Omega \times(a, b))$.

Proof. We follow the proof of in [9, Theorem 3.1]. D is used to denote the algebra generated by all open cubes in $R^{n+1}$ with rational vertices and $\mathrm{E}$ the class of all bounded open sets in $R^{n+1}$. Applying lemma 2.2 and a diagonalization argument, we can find a sequence $\varepsilon_{h} \quad(h \rightarrow \infty)$ such that $\Gamma(\tau) \lim _{h \rightarrow \infty} F^{\varepsilon_{h}}(u, Q)$ exists for all $Q \in D$, i.e

$$
H^{-}(u, Q)=H^{+}(u, Q), \quad \forall Q \in D,
$$

where

$$
H^{-}(u, Q)=\Gamma^{-}(\tau) \lim _{h \rightarrow \infty} F^{\varepsilon_{h}}(u, Q)
$$


and

$$
H^{+}(u, Q)=\Gamma^{+}(\tau) \lim _{h \rightarrow \infty} F^{\varepsilon_{h}}(u, Q) .
$$

In the same way as in [9, p.738-739], by lemma B in [6], we can prove that $H^{-}$is (finitely) super-additive and $H^{+}$is sub-additive over D. For $e \in E$, define

$$
H(u, e)=\sup _{Q \subset \subset e} H^{-}(u, Q)=\sup _{Q \subset \subset e} H^{+}(u, Q) \quad Q \in D
$$

then $H(u, e)$ is an increasing, inner regular and finitely additive set function. Therefor, the routine methods implies that (2.4) holds and $H(u, \Omega \times(a, b))$ can be extended to a $\sigma$-finite and $\sigma$-additive measure on $\beta_{T}$ (see [11, Prop. 5.5 and Theorem 5.6 ]. From (2.4) and (1.5), the estimate (2.5) follows immediately .

\section{3. Г-LIMITS OF LAYERED AFFINE FUNCTIONS}

Throughout this section, suppose that (1.4), (1.5) and (1.8) are satisfied. $\tau$ is used to denote the sw-topology of $V_{p}\left(\Omega_{T}, m\right)$. For simiplicity, $V_{p}\left(\Omega_{T}\right)$ denotes the space $V_{p}\left(\Omega_{T}, m\right)$. We intend to determine the $\Gamma$-limits of $F^{\varepsilon}\left(u, \Omega_{T}\right)$ for $u=$ $\lambda(t) \cdot x+a(t)$ with $\lambda \in L^{p}([0, T], M(m \times n))$ and $a \in L^{p}\left([0, T], R^{m}\right)$, where we define the norm on $M(m \times n)$, the space of all real $m \times n$ matrices, as the same as on $R^{m n}$.

LEMMA 3.1. For each $u_{\lambda, a}=\lambda(t) \cdot x+a(t)$ with

$$
\lambda \in L^{p}([0, T], M(m, n)) \text { and } a \in L^{p}\left([0, T], R^{m}\right),
$$

there exists a sequence of functions $\left\{u^{\varepsilon}\right\} \subset V_{p}\left(\Omega_{T}\right)$ satifying

$$
\left\{u^{\varepsilon}-u_{\lambda, a}\right\} \subset V_{p}^{0}\left(\Omega_{T}\right) \text { and } u^{\varepsilon} \stackrel{\tau}{\rightarrow} u_{\lambda, a} \text { in } V_{p}\left(\Omega_{T}\right) \text { as } \varepsilon \rightarrow 0^{+}
$$

such that

$$
\lim _{\varepsilon \rightarrow 0^{+}} F^{\varepsilon}\left(u^{\varepsilon}, \Omega_{T}\right)=\int_{\Omega_{T}} \bar{f}(t, \lambda) d x d t=F\left(u_{\lambda, a}, \Omega_{T}\right)
$$

where $\bar{f}(t, \lambda)$ is given by (1.6) and $F$ by (1.7).

Proof . Fix $\delta \in(0,1)$, one can choose $k \in N$ and $\phi^{\delta} \in V_{p}^{0}\left(k_{T}, m\right)$ (see (1.6)) such that

$$
\bar{f}(t, \lambda(t)) \leq|k Y|^{-1} \int_{k Y} f\left(y, t, \lambda+D \phi^{\delta}\right) d y \leq \bar{f}(t, \lambda(t))+\delta .
$$

We use $E_{\eta}^{*}$ to denote the extension of $\eta \bar{Y}$ on the $\eta Y$-period, and let

$$
\Omega_{\eta}^{*}=\left\{e \in E_{\eta}^{*}, e \subset \Omega\right\}, \quad E_{\eta}=\bigcup_{e \in E_{\eta}^{*}} e, \quad \Omega_{\eta}=\bigcup_{e \in \Omega_{\eta}^{*}} e
$$


then $E_{\eta}=R^{n}$. As $\Omega$ is bounded, $\Omega_{\eta}^{*}$ is a finite set for each $\eta>0$, and

$$
\lim _{\eta \rightarrow 0^{+}} L^{n}\left(\Omega \backslash \Omega_{\eta}\right)=0 .
$$

For every $t \in[0, T]$, extend $\phi^{\delta}(y, t)$ such that it is a $k Y$-periodic function on the variable $\mathrm{y}$, then define

$$
v^{\varepsilon, \delta}(x, t)=\left\{\begin{array}{ll}
u_{\lambda, a}(x, t)+\varepsilon \phi^{\delta}\left(\frac{x}{\varepsilon}, t\right), & \Omega_{\varepsilon k} \\
u_{\lambda, a}(x, t), & \Omega \backslash \Omega \varepsilon k
\end{array} .\right.
$$

It is easy to know that $v^{\varepsilon, \delta} \in V_{p}\left(\Omega_{T}\right), v^{\varepsilon, \delta}-u_{\lambda, a} \in V_{p}^{0}\left(\Omega_{T}\right)$. For each $D \in \Omega_{\varepsilon k}$, by the periodicity of

$$
g(y, t)=f\left(y, t, \lambda(t)+D \phi^{\delta}(y, t)\right)
$$

we have

$$
\begin{gathered}
\int_{D \times(0, T)} f\left(\frac{x}{\varepsilon}, t, D v^{\varepsilon, \delta}\right) d x d t=\int_{0}^{T}\left[\varepsilon^{n} \int_{D / \varepsilon} f\left(y, t, \lambda(t)+D \phi^{\delta}(y, t) d y\right] d t\right. \\
=L^{n}(D) \int_{0}^{T} d t|k Y|^{-1} \int_{k Y} f\left(y, t, \lambda(t)+D \phi^{\delta}\right) d y
\end{gathered}
$$

Summing up the both sides for all $D \in \Omega_{\varepsilon k}$ and applying (3.1), we obtain that

$$
\begin{aligned}
L^{n}\left(\Omega_{\varepsilon k}\right) \int_{0}^{T} \bar{f}(t, \lambda(t)) d t & \leq \int_{0}^{T} d t \int_{\Omega_{\varepsilon k}} f\left(\frac{x}{\varepsilon}, t, D v^{\varepsilon, \delta}\right) d x \\
& \leq L^{n}\left(\Omega_{\varepsilon k}\right) \int_{0}^{T}(\bar{f}(t, \lambda(t))+\delta) d t .
\end{aligned}
$$

Thus, it follows from (1.5) and (3.3) that

$$
\begin{aligned}
& L^{n}\left(\Omega_{\varepsilon k}\right) \int_{0}^{T} \bar{f}(t, \lambda(t)) d t \leq \int_{\Omega_{T}} f\left(\frac{x}{\varepsilon}, t, D v^{\varepsilon, \delta}\right) d x d t \\
& \leq L^{n}\left(\Omega_{\varepsilon k}\right) \int_{0}^{T}(\bar{f}(t, \lambda(t))+\delta) d t+\int_{0}^{T} d t \int_{\Omega \backslash \Omega_{\varepsilon k}} f\left(\frac{x}{\varepsilon}, t, \lambda(t)\right) d x \\
& \leq L^{n}\left(\Omega_{\varepsilon k}\right) \int_{0}^{T}(\bar{f}(t, \lambda(t))+\delta) d t+C L^{n}\left(\Omega \backslash \Omega_{\varepsilon k}\right) \int_{0}^{T}\left(1+|\lambda|^{p}\right) d t .
\end{aligned}
$$

By this estimate and (3.2), we see that

$$
\lim _{\delta \rightarrow 0^{+}} \lim _{\varepsilon \rightarrow 0^{+}} \int_{\Omega_{T}} f\left(\frac{x}{\varepsilon}, t, D v^{\varepsilon, \delta}\right) d x d t=\int_{\Omega_{T}} \bar{f}(t, \lambda(t)) d t d x .
$$


Moreover, we have

$$
\left\|v^{\varepsilon, \delta}-u_{\lambda, a}\right\|_{L^{p}\left(\Omega_{T}\right)}^{p}=\varepsilon^{p} \sum_{D \in \Omega_{\varepsilon k}}|D| \int_{0}^{T} d t|k Y|^{-1} \int_{k Y}\left|\phi^{\delta}\right|^{p} d y
$$

Applying (3.6), (3.7), and lemma 2.4, one can find a sequence

$$
\delta(\varepsilon) \rightarrow 0^{+} \quad \text { as } \varepsilon \rightarrow 0^{+}
$$

such that $\left\{u^{\varepsilon}=v^{\varepsilon, \delta(\varepsilon)}: \varepsilon>0\right\}$ satisfy that

$$
\left\{u^{\varepsilon}-u_{\lambda, a}\right\} \subset V_{p}^{0}\left(\Omega_{T}\right), \quad \lim _{\varepsilon \rightarrow 0^{+}}\left\|u^{\varepsilon}-u_{\lambda, a}\right\|_{L^{p}\left(\Omega_{T}\right)}^{p}=0
$$

and

$$
\lim _{\varepsilon \rightarrow 0^{+}} F^{\varepsilon}\left(u^{\varepsilon}, \Omega_{T}\right)=F\left(u_{\lambda, a}, \Omega_{T}\right) .
$$

On the other hand, the coercive condition in (1.5) and (3.5) imply that $\left\{D u^{\varepsilon}\right\}$ is bounded in $L^{p}\left(\Omega_{T}, R^{m n}\right)$. Thus, by lemma B in [6], we obtain that $u^{\varepsilon} \stackrel{\tau}{\rightarrow} u_{\lambda, a}$. This proves the desired result.

LEMMA 3.2. Let $u_{\lambda, a}(x, t)=\lambda(t) \cdot x+a(t)$ be the same as in lemma 3.1. Then for each sequence $u^{\varepsilon} \stackrel{\tau}{\rightarrow} u_{\lambda, a}$ in $V_{p}\left(\Omega_{T}\right) \quad\left(\varepsilon \rightarrow 0^{+}\right)$,

$$
\liminf _{\varepsilon \rightarrow 0^{+}} F^{\varepsilon}\left(u^{\varepsilon}, \Omega_{T}\right) \geq F\left(u_{\lambda, a}, \Omega_{T}\right)=\int_{\Omega_{T}} \bar{f}(t, \lambda(t)) d t d x .
$$

Proof . (1) Firstly, assume $u^{\varepsilon} \stackrel{\tau}{\rightarrow} u_{\lambda, a}$ and $u^{\varepsilon}-u_{\lambda, a} \in V_{p}^{0}\left(\Omega_{T}\right)$. As $\Omega$ is bounded, we find an open cube $\mathrm{D}$ whose sides are parallel to axes and whose center concides with the origin, such that $\bar{\Omega} \subset D$. The side length of $\mathrm{D}$ is denoted by $2 \mathrm{~d}$, and let

$$
\begin{gathered}
k_{\varepsilon}=\left[\frac{2 d}{\varepsilon}\right]+3, \quad a_{\varepsilon}=\left[-\frac{d}{\varepsilon}\right], \\
x_{\varepsilon}=\left(a_{\varepsilon}, \cdots, a_{\varepsilon}\right) \in R^{n}, D_{\varepsilon}=\varepsilon\left(x_{\varepsilon}+k_{\varepsilon} Y\right),
\end{gathered}
$$

where $[\kappa]$ denote the maximum integer not greater than $\kappa$. It is not difficult to get

$$
D \subset D_{\varepsilon}, \quad \lim _{\varepsilon \rightarrow 0^{+}} L^{n}\left(D_{\varepsilon}\right)=L^{n}(D) .
$$

Let

$$
Q=D \backslash \bar{\Omega}, \quad Q_{T}=Q \times(0, T) .
$$

Applying lemma 3.1 to the open set $Q$, we can choose a sequence

$$
v^{\varepsilon} \rightarrow u_{\lambda, a} \quad \text { sw in } \quad V_{p}\left(Q_{T}\right), \quad v^{\varepsilon}-u_{\lambda, a} \in V_{p}^{0}\left(Q_{T}\right)
$$


such that

$$
\lim _{\varepsilon \rightarrow 0^{+}} F^{\varepsilon}\left(v^{\varepsilon}, Q_{T}\right)=\int_{Q} \bar{f}(t, \lambda) d x d t .
$$

For fixed $t \in[0, T]$, define

$$
\phi^{\varepsilon}(x, t)= \begin{cases}u^{\varepsilon}-u_{\lambda, a}, & x \in \bar{\Omega} \\ v^{\varepsilon}-u_{\lambda, a}, & x \in D \backslash \bar{\Omega}=Q . \\ 0, & x \in D_{\varepsilon} \backslash D\end{cases}
$$

By the periodicity of $f(y, t, \lambda)$, using the variable transformation, we obtain

$$
\begin{gathered}
\int_{D_{\varepsilon}} f\left(\frac{x}{\varepsilon}, t, \lambda+D \phi^{\varepsilon}(x, t)\right) d x=\varepsilon^{n} \int_{x_{\varepsilon}+k_{\varepsilon} Y} f\left(y, t, \lambda+D_{x} \phi^{\varepsilon}(\varepsilon y, t)\right) d y \\
\quad=\left(k_{\varepsilon} \varepsilon\right)^{n}\left|k_{\varepsilon} Y\right|^{-1} \int_{k_{\varepsilon} Y} f\left(y, t, \lambda+D \psi^{\varepsilon}(y, t)\right) d y
\end{gathered}
$$

where $\psi^{\varepsilon}(y, t)=\varepsilon^{-1} \phi^{\varepsilon}\left(\varepsilon\left(y+x_{\varepsilon}\right), t\right)$. Obviously, (3.11) gives us

$$
\psi^{\varepsilon} \in V_{p}^{0}\left(\left(k_{\varepsilon} Y\right) \times(0, T)\right) .
$$

Thus, we deduce, from (3.12) and (1.6) yield that for each $t \in[0, T]$,

$\left|D_{\varepsilon}\right|^{-1} \int_{D_{\varepsilon}} f\left(\frac{x}{\varepsilon}, t, \lambda+D \phi^{\varepsilon}(x)\right) d x=\left|k_{\varepsilon} Y\right|^{-1} \int_{K_{\varepsilon} Y} f\left(y, t, \lambda+D \psi^{\varepsilon}(y, t)\right) d y \geq \bar{f}(t, \lambda)$.

Therefore

$$
\int_{0}^{T} \int_{D_{\varepsilon}} f\left(\frac{x}{\varepsilon}, t, \lambda+D \phi^{\varepsilon}\right) d x d t \geq L^{n}\left(D_{\varepsilon}\right) \int_{0}^{T} \bar{f}(t, \lambda) d t .
$$

On the other hand, by (1.5) and (3.8), we have

$$
\liminf _{\varepsilon \rightarrow 0^{+}} \int_{0}^{T} d t \int_{D_{\varepsilon}} f\left(\frac{x}{\varepsilon}, t, \lambda+D \phi^{\varepsilon}\right) d x d t=\liminf _{\varepsilon \rightarrow 0^{+}} \int_{0}^{T} d t \int_{D} f\left(\frac{x}{\varepsilon}, t, \lambda+D \phi^{\varepsilon}\right) d x .
$$

This yields

$$
\liminf _{\varepsilon \rightarrow 0^{+}} F^{\varepsilon}\left(u_{\lambda, a}+\phi^{\varepsilon}, D \times(0, T)\right) \geq \int_{0}^{T} \int_{D} \bar{f}(t, \lambda) d x d t .
$$

Combing this estimate, (3.9), (3.10) with (3.11), we have

$$
\begin{aligned}
\liminf _{\varepsilon \rightarrow 0^{+}} F^{\varepsilon}\left(u^{\varepsilon}, \Omega_{T}\right) & =\liminf _{\varepsilon \rightarrow 0^{+}}\left[F^{\varepsilon}\left(u_{\lambda, a}+\phi^{\varepsilon}, D \times(0, T)\right)-F^{\varepsilon}\left(v^{\varepsilon}, Q \times(0, T)\right)\right] \\
& \geq \int_{0}^{T} \int_{D} \bar{f}(t, \lambda) d x d t-\int_{0}^{T} \int_{Q} \bar{f}(t, \lambda) d x d t \\
& =\int_{\Omega_{T}} \bar{f}(t, \lambda) d x d t .
\end{aligned}
$$


(2) In order to remove the restriction $u^{\varepsilon}-u_{\lambda, a} \in V_{p}^{0}\left(\Omega_{T}\right)$, it is sufficient to apply the De Giorgi's arguements and the result of the case (1). See [11], or [8, p.197] for the details.

DEFINITION 3.3. Let $\left\{\Omega_{i}: i=1,2, \cdots h\right\}$ be a finite partition of $\Omega$ into open sets (except for a set of measure zero ), $\lambda_{i} \in L^{p}([0, T], M(m, n)), \quad a_{i} \in$ $L^{p}\left([0, T], R^{m}\right)$. We call the function

$$
W(x, t)= \begin{cases}\lambda_{i}(t) \cdot x+a_{i}(t), & x \in \Omega_{i} \\ 0, & x \in \Omega \backslash \cup_{i=1}^{h} \Omega_{i}\end{cases}
$$

a $L^{p}$-layered affine function on $\Omega_{T}$.

To sum up lemmas 3.1 and 3.2 (observing that $\Omega$ maybe arbitrary there ), lemmas 2.5 and 2.3 , we obtain the following theorem.

THEOREM 3.4. Suppose that $\Omega$ is a bounded open set in $R^{n}$ with $L^{n}(\partial \Omega)$ $=0, H\left(u, \Omega_{T}\right)$ is given by lemma 2.5 , then

$$
\Gamma(\tau) \lim _{\varepsilon \rightarrow 0^{+}} F^{\varepsilon}\left(w, \Omega_{T}\right)=\int_{\Omega_{T}} \bar{f}(t, D w) d x d t=H\left(w, \Omega_{T}\right)
$$

for any $w$, a $L^{p}$-layered affine function on $\Omega_{T}$.

\section{A PROOF OF THEOREM 1.2}

In this section, we suppose that all the hypotheses of Theorem 1.2 are satisfied. Applying the same arguement as in [9, Section 5], we can prove that for almost $t \in[0, T], \bar{f}(t, \lambda)$ is convex if $n=2$; and convex with respect to each column vector if $n>2$. This implies that

LEMMA 4.1. For a.e. $t \in[0, T], \bar{f}(t, \lambda)$ is continuous in $M(m, n)$.

LEMMA 4.2. Suppose $v \in V_{p}\left(\Omega_{T}\right) \quad(1<p<\infty)$, then there exists a sequence of $L^{p}$-layered affine functions :

$$
v^{k}(x, t)= \begin{cases}\lambda_{i}^{k}(t) \cdot x+a_{i}^{k}(t), & x \in \Omega_{i} \\ 0, & x \in \Omega \backslash \cup_{i=1}^{h_{k}} \Omega_{i}\end{cases}
$$

such that $\left\|v-v^{k}\right\|_{V_{p}\left(\Omega_{T}\right)} \longrightarrow 0 \quad$ as $\quad k \rightarrow \infty$.

Proof . (1) Suppose $1<p \leq 2$. Fix $v \in V_{p}\left(\Omega_{T}\right)$. For any $\varepsilon>0$ we can choose $u \in V_{2}\left(\Omega_{T}\right)$ such that

$$
\|u-v\|_{V_{p}\left(\Omega_{T}\right)}<\varepsilon .
$$

Because $H \stackrel{\text { def }}{=} W^{1,2}(\Omega)$ is a Hilbert space, one can assume that $\left\{\psi_{l}\right\}_{l=1}^{\infty}$ is its complete orthonormal basis. Let

$$
C_{l}(t)=<u(t, \cdot), \psi_{l}>_{H},
$$


then $C_{l}(t) \in L^{2}[0, T]$, and for a.e. $t \in[0, T]$

$$
I_{k}(t)=\left\|u-\sum_{l=1}^{k} C_{l} \psi_{l}(x)\right\|_{H} \longrightarrow 0 \quad(k \rightarrow \infty)
$$

Thus the domainnated convergence theorem implies that for some integer $\mathrm{k}$

$$
\left\|u-\sum_{l=1}^{k} C_{l} \psi_{l}\right\|_{V_{p}\left(\Omega_{T}\right)} \leq \varepsilon .
$$

It is well known that there exist piecewise affine functions $\omega_{l}(x)$ in $\Omega$ such that

$$
\max _{1 \leq l \leq k}\left\|\psi_{l}-\omega_{l}\right\|_{W^{1, p}(\Omega)} \leq \varepsilon\left(1+\sum_{l=1}^{k}\left\|C_{l}\right\|_{L^{p}(\Omega)}^{-1}\right) .
$$

Let

$$
v^{\varepsilon}(x, t)=\sum_{l=1}^{k} C_{l}(t) \omega_{l}(x)
$$

then

$$
\left\|v^{\varepsilon}-\sum_{l=1}^{k} C_{l} \psi_{l}\right\|_{V_{p}\left(\Omega_{T}\right)} \leq C(p) \varepsilon .
$$

Combing (4.1), (4.2) with (4.3), we get

$$
\left\|v-v^{\varepsilon}\right\|_{V_{p}\left(\Omega_{T}\right)} \leq C(m, n, p) \varepsilon .
$$

Observing that each $v^{\varepsilon}$ can be written as a layered function on $\Omega_{T}$, we have completed the proof.

(2) Suppose $2<p<\infty$. Applying Sobolev embedding theorem we can find an integer $k, \frac{k-1}{n} \geq \frac{1}{2}-\frac{1}{p}$, such that

$$
H_{1} \stackrel{\text { def }}{=} W^{k, 2}(\Omega) \hookrightarrow W^{1, p}(\Omega) .
$$

Given $v \in V_{p}\left(\Omega_{T}\right)$. For $\varepsilon>0$, one can find $u \in L^{p}\left([0, T], W^{k, 2}(\Omega)\right)$ such that

$$
\|u-v\|_{V_{p}\left(\Omega_{T}\right)}<\varepsilon .
$$

Let $\left\{\psi_{l}\right\}_{l=1}^{\infty}$ be the complete orthonormal basis of the Hilbert space $H_{1}$, then

$$
C_{l}(t) \stackrel{\text { def }}{=}<u(\cdot, t), \psi_{l}>_{H_{1}} \in L^{p}[0, T] .
$$

The remaining part is entirely the same as the case (1). 
Now we are in the position to prove Theorem 1.5. We will use the idea of [9, p.750-751]. For $u \in V_{p}\left(\Omega_{T}\right)$, we can extend $u$ such that $u \in V_{p, l o c}$ (recall (2.3) ). From lemma 4.2, choose a sequence of $L^{p}$-layered functions $\omega^{k}(x, t)$, such that

$$
\left\|u-\omega^{k}\right\|_{V_{p}\left(\Omega_{T}\right)} \longrightarrow 0 \quad(k \rightarrow \infty) .
$$

By taking a subsequence, one can assume that $D \omega^{k} \rightarrow D u$ almost everywhere on $\Omega_{T}$ and

$$
\bar{f}\left(t, D \omega^{k}\right) \longrightarrow \bar{f}(t, D u) \text { a.e in } \Omega_{T}
$$

by the virtue of the continuity of $\bar{f}(t, \cdot)$ (see Lemma 4.1 ).

We deduce, from the absolute continuity of $\int|D u|^{p} d x d t$, Egoroff theorem, theorem 3.4 and inequality (2.5), that

$$
\liminf _{k \rightarrow \infty} \int_{\Omega_{T}} \bar{f}\left(t, D \omega^{k}\right) d x d t \leq \int_{\Omega_{T}} \bar{f}(t, D u) d x d t .
$$

Therfore, by the semi-continuity of $H\left(u, \Omega_{T}\right)$ (see lemma 2.1 ),

$$
\begin{aligned}
H\left(u, \Omega_{T}\right) & \leq \liminf _{k \rightarrow \infty} H\left(\omega^{k}, \Omega_{T}\right) \\
& =\liminf _{k \rightarrow \infty} \int_{\Omega_{T}} \bar{f}\left(t, D \omega^{k}\right) d x d t \\
& \leq \int_{\Omega_{T}} \bar{f}(t, D u) d x d t .
\end{aligned}
$$

On the other hand, according to lemma 2.5 and Lebesgue-Nikodym theorem (see $\S 3$ of Ch.3 in [12] ), we have

$$
H\left(u, \Omega_{T}\right)=\int_{\Omega_{T}} h(x, t) d x d t
$$

for some $h \in L_{l o c}^{1}\left(R^{n} \times(0, T)\right)$ and all $\Omega_{T}=\Omega \times(0, T)$. By approximation argument, one can easily prove that for a.e $(x, t) \in \Omega_{T}$, there exists $r_{k} \rightarrow 0^{+}$such that

$$
\frac{u\left(x+r_{k}(y-x), t\right)-u(x, t)}{r_{k}} \stackrel{\tau}{\rightarrow} D u(x, t) \cdot(y-x) \quad \text { in } \quad V_{p}(B(x, 1) \times(0, T)) .
$$

Since

$$
\begin{aligned}
& \left.\left|\int_{0}^{T} h(x, t) d t-\int_{0}^{T}\right| B\left(x, r_{k}\right)\right|^{-1} \int_{B\left(x, r_{k}\right)} h(y, t) d y d t \mid \\
& \quad \leq\left|B\left(x, r_{k}\right)\right|^{-1} \int_{B\left(x, r_{k}\right)}\left|\int_{0}^{T}[h(x, t)-h(y, t)] d t\right| d y
\end{aligned}
$$


and

$$
\int_{0}^{T} h(y, t) d t \in L_{l o c}^{1}\left(R^{n}\right)
$$

so

$$
\int_{0}^{T} h(x, t) d t=\lim _{k \rightarrow \infty} \int_{0}^{T}\left|B\left(x, r_{k}\right)\right|^{-1} \int_{B\left(x, r_{k}\right)} h(y, t) d y d t \quad \text { for a.e. } x \in \Omega .
$$

Fix $k$ and $x$, set

$$
r=r_{k}, \quad B_{r}=B(x, r), \quad B_{r, T}=B_{r} \times(0, T) .
$$

By (4.7) and lemma 2.5, we can find a sequence

$$
u^{h} \stackrel{\tau}{\rightarrow} u \text { in } V_{p}\left(B_{r, T}\right)(h \rightarrow 0)
$$

such that

$$
\begin{aligned}
\int_{0}^{T}\left|B_{r}\right|^{-1} \int_{B_{r}} h(y, t) d y d t=\left|B_{r}\right|^{-1} H\left(u, B_{r, T}\right) \\
\quad=\lim _{h \rightarrow \infty} \int_{0}^{T}\left|B_{r}\right|^{-1} \int_{B_{r}} f\left(\frac{y+\varepsilon_{h} k_{h}}{\varepsilon_{h}}, t, D u^{h}\right) d y d t, \quad\left(k_{h} \stackrel{\text { def }}{=}\left[\frac{x(r-1)}{\varepsilon_{h}}\right]\right) \\
\geq \liminf _{h \rightarrow \infty} \int_{0}^{T}\left|B_{\frac{r}{2}}\right|^{-1} \int_{B_{\frac{r}{2}}} f\left(\frac{y+x(r-1)}{\varepsilon_{h}}, t, D u^{h}\left(y+a_{h}, t\right)\right) d y d t \\
\quad\left(\text { note } t h a t \quad a_{h} \stackrel{\text { def }}{=} x(r-1)-\varepsilon_{h} k_{h} \rightarrow 0^{+}\right) \\
=\liminf _{h \rightarrow \infty} \int_{0}^{T}\left|B_{\frac{1}{2}}\right|^{-1} \int_{B_{\frac{1}{2}}} f\left(\frac{r y}{\varepsilon_{h}}, t, D\left(u^{h}\left(x+r(y-x)+a_{h}, t\right)\right.\right. \\
\left.\quad-u(x, t)) r^{-1}\right) d y d t .
\end{aligned}
$$

Let $u_{r, x}(y, t)=r^{-1}[u(x+r(y-x), t)-u(x, t)]$. Obviously

$$
r^{-1}\left[u^{h}\left(x+r(y-x)+a_{h}, t\right)-u(x, t)\right] \stackrel{\tau}{\rightarrow} r^{-1} u_{r, x} \quad \text { in } \quad V_{p}\left(B_{\frac{1}{2}, T}\right) \quad \text { as } \quad h \rightarrow \infty .
$$

Let

$$
\delta_{h}=r^{-1} \varepsilon_{h}, \quad a=\left|B_{\frac{1}{2}}\right|^{-1}, \quad F^{-}(u, Q)=\Gamma^{-}(\tau) \lim _{h \rightarrow \infty} F^{\delta_{h}}(u, Q) .
$$

By (4.10), lemmas 2.1 and 2.3, (4.8) and theorem 3.4 in that order, we deduce that

$$
\begin{aligned}
\lim _{k \rightarrow \infty} \int_{0}^{T}\left|B\left(x, r_{k}\right)\right|^{-1} \int_{B\left(x, r_{k}\right)} h(y, t) d y d t & \geq a \cdot \liminf _{k \rightarrow \infty} F^{-}\left(u_{r_{k}, x}, B_{\frac{1}{2}, T}\right) \\
& \geq a \cdot F^{-}\left(D u(x, t)(y-x), B_{\frac{1}{2}, T}\right) \\
& =a \int_{0}^{T} \int_{B\left(x, \frac{1}{2}\right)} \bar{f}(t, D u(x, t) d t d y \\
& =\int_{0}^{T} \bar{f}(t, D u(x, t)) d t .
\end{aligned}
$$


Combing this estimate with (4.9), we obtain

$$
\int_{\Omega_{T}} h(x, t) d x d t \geq \int_{\Omega_{T}} \bar{f}(x, D u) d x d t
$$

which together with (4.7) implies the opposite inequality of (4.6). Hence

$$
H\left(u, \Omega_{T}\right)=\int_{\Omega_{T}} \bar{f}(t, D u) d x d t \quad \forall u \in V_{p}\left(\Omega_{T}\right) .
$$

Observing that

$$
F\left(u, \Omega_{T}\right)=\int_{\Omega_{T}} \bar{f}(t, D u) d x d t
$$

is independent of $\left\{\varepsilon_{h}\right\}$, we have completed the proof of Theorem 1.2 by lemma 2.5 .

\section{REFERENCES}

[1] H. Attouch, Variational convergence for functions and operators, Appl. Math. Series, Pitman, 1984 .

[2] G. Dal Maso, An introduction to $\Gamma$-convergence, Boston Basel: Birkhäuser, 1993.

[3] E. De Giorgi, New problems in $\Gamma$-convergence and G-convergence in Free Boundary Problems, Proc. of seminar held in Pavia, September-October 1979, Ist. Naz. Alt. Mat. Francesco Severi, Vol. II, 183-194, Rome, 1980.

[4] L. Bronsard and R.V. Kohn, Motion by mean curvature as the singular limit of Ginzburg-Landau Dynamics, J. Differ. Eqn. 90 (1991), 211-237.

[5] N. C. Owen, J. Rubinstein and P. Sternberg, Minimizers and gradient flows for singularly perturbed bi-stable potentials with a Dirichlet condition, Proc. R. Soc. Lond Ser.A 429 (1990), 505-532.

[6] H.Y. Jian, A relation between $\Gamma$-convergence of functionals and their associated gradient flows, Science in China, Ser. A, 42(2)(1999), 133-139.

[7] H.Y. Jian, Homogenization problems of parabolic minima, Acta Math. Appl. Sinica 12 (1996), 318-327.

[8] S. Müller, Homogenization of noncovex integral functionals and elastic materials, Arch. Rat. Mech. Anal. 99 (1987), 187-212.

[9] E. Weinan, A class of homogenization problems in the calculus of variations, Commu. pure Appl. Math. 44 (1991), 733-759.

[10] H.Y. Jian, $\Gamma$-convergence of noncoercive functionals in vector-valued space $W^{1, p}(\Omega)$ (in chinese), Science in China Ser. A 24(3) (1994), 233-240.

[11] E. De Giorgi and G. Letta, Une notion general de convergence faible pour des fontions crotssantes d'ensemble, Ann Scuola Norm. Sup. Pisa CI Sci. 4 (1977), 61 -99.

[12] K. Yosida, Functional analysis, 5th Ed, Springer-Verlag, 1975. 